\title{
Context Network
}

\author{
Hooman Tahayori ${ }^{1}$, Giovanni Degli Antoni ${ }^{1}$, Elena Pagani ${ }^{2}$, Sadegh Astaneh ${ }^{2}$ \\ ${ }^{1}$ Universita degli Studi di Milano, Dipartimento di Scienze dell'Informazione, \\ ${ }^{2}$ Universita degli Studi di Milano, Dipartimento di Informatica e Comunicazione, \\ ${ }^{1,2}$ Via Comelico 39/41, 20135, Milano, MI, Italy \\ hooman.tahayori@unimi.it,gda@dsi.unimi.it,pagani@dico.unimi.it, sadegh.astaneh@unimi.it
}

\begin{abstract}
Context network is the result of the integration of Wireless Sensor Network (WSN) and context-awareness which are primarily reconsidered in terms of granular computing. It is aimed to be reliable infrastructure for providing applicable and proper contextual information necessary for developing realistic context-aware applications.
\end{abstract}

Keywords-component; Context Network; Wireless Sensor Network (WSN); Granular Computing;

\section{INTRODUCTION}

Context-aware applications, either reactive or proactive, in order to work properly, must be fed with reliable, certain and appropriate contextual information. There has been considerable efforts on providing frameworks and models for designing context-aware applications per se, but rare on gathering contextual information. What is common among most of the papers on context-aware applications is either the assumption of the availability of contextual information or the existence of some special sensors for gathering contextual information. But some important questions like How to choose proper sensors? How to deploy sensors? How to integrate sensors to the applications? How to draw different abstraction from raw data? How to reuse sensors simultaneously? Cost of the context-gathering sensors? and more, are not answered. Moreover, providing raw numerical data from environment for applications, may not be that helpful since "context information is useful only when it can be usefully interpreted, and it must be treated with sensitivity" [15]. It is imaginable that context-aware applications be blurred into other kinds of applications; to clarify we remind that interacting with an application would be either by an explicit act of communication (e.g. typing on keyboard) or implicitly that is context.

Contextual information is mainly the description of the world of an entity, which is definitely far beyond simple numerical data. Rather, contextual information is an aggregated, interpreted and abstraction of similar, coherence, functional adjacent numerical data or in other words an information granule as defined in [2] that enables users "building efficient and user-centered views of the external world and supporting and facilitating [their] perception of the surrounding physical and virtual world" [2]. Hence, it is natural to treat contextual information in terms of granular computing which provides an efficient and applicable view towards contextual information.

In [19] it is revealed that for the specified application, one sensor does not suffice and combination of multiple sensors is required. Traditionally, sensors are either, expensive, large and complex systems that are deployed far away from the phenomena to be sensed or are carefully engineered into a network that can only transmit time series of the sensed phenomena to other nodes with computational capabilities [12]. However, recent advancement in the fields of wireless communication, digital electronics and microelectromechanical-systems (MEMS) have enabled the development of Wireless Sensor Networks (WSN) build up of low-power, cheap, multifunctional and small in size sensors equipped with sensing, data processing and communicating components, and are supposed to be used unattendedly, even in the environments where the direct presence of users is not expedient. These sensors are not as reliable or as accurate as their counterpart macrosensors, but their price, dimension, communication ability along with network protocols with self organizing capabilities, enable simply deploying and networking hundreds to thousands of them and expect accurate and reliable data. Potential advantages of WSNs over other architectures would be counted as ensuring greater signal to noise ratio, greater fault tolerance, coverage of a very large area, improving sensing performance by including multiple sensor types, overcoming environmental effects, localizing discrete phenomena, rapid and cost effective deployment, self-organizing, communicating compressed data, robust against nodes' failure, scalable [9], [17]. WSN, as a key technology of the $21^{\text {st }}$ century [14], in essence, "will provide the end user with intelligence and better understanding of the environment" [1]. However, it is apparent that to realize the claim, integration of granular computing with WSN is unavoidable.

The aim of this paper is to integrate WSN, contextawareness and granular computing to drive a framework, Context Network, that provides reliable,certain and proper contextual information in different levels of granularity. This will ease developing context-aware applications mainly due to the availability of reliable and appropriate contextual information. 


\section{CONTEXT AWARENESS}

In literature, most of the definitions for context, are by example which results in non-general definitions. The one that seems to be general enough and said to be more applicable is: "context is any information that can be used to characterize the situation of an entity. An entity is person, place, object that is considered relevant to the interaction between a user and an application, including an application and the user themselves"'[6]. By this definition contextual information can range from identity, spatial information, temporal information to physiological measurements, social situation, history of interaction, and even happiness, dangerous and critical situations. It is clear that not all the contextual information would be sensed directly, rather, must be derived from other directly sensible contexts which requires namely super sensors [4] to be devised.

Some context-aware applications yet exist however almost all of them are developed in research labs. As an example, the Active Badge [15], conference assistance [8], GUIDE [5] and In/Out Board [18] would be enumerated. A considerable point in existing context-aware applications is that, all of them are based on simple, well-defined and easily sensible contextual information (mainly at the numerical data level) and moreover on the assumption of the existence of mechanisms for gathering contextual information. It has had its impact on the applications by (i) limiting the applications to the just sensible context that might not be that appropriate and reliable for the application, (ii) limiting the diversity of context-aware applications, (iii) limiting access to the higher level contextual information specially those that are not directly sensible, (iv) limiting applications to specified context sensing infrastructures and (v) limiting context to predefined spots of the environment.

Numerical data, gathered from environment, constitute finest contextual information and are not necessarily helpful. However, their aggregates, based on their similarity, functionality, proximity, coherency, and indistinguishability and alike, make more sense and would be more helpful, e.g. position of a person in the form of e.g. $(x, y, z)$ does not make that sense as "he is in his office" in response to "where is the boss?". What is definitely required is the existence of a hierarchical, extensible and realistic view towards the contextual information. Towards the aim, given the domain of $X$, we define granular plane as a four-tuple $\psi_{i}^{k}=\left(X, G^{k}, A_{i}^{k}, C_{i}^{k}\right)$, where $G^{k}$ is a formal framework of granulation of the $k$ th hierarchy, $A_{i}^{k}$ constitutes covering set of related frames of reference at the $i$ th level and $C_{i}^{k}$ denotes communication rules to lower or upper granular planes in the same hierarchy. Consequently $\psi_{i}^{k}$ denotes $i$ th granular plane in the kth hierarchy. Granular hierarchy, on the other hand is a hierarchy of granular planes with the same formal framework and granulation criterion defined as $\Psi^{k}=\left(X, \psi^{k}, R^{k}\right)$ where $\psi^{k}=\bigcup_{i=1} \psi_{i}^{k}, \quad R^{k}$ denotes the granulation criterion of the $k$ th granular hierarchy. Granular plane $\psi_{i}^{k}$ in the granular hierarchy $\Psi^{k}$ is said to be finer than $\psi_{j}^{k}$ if and only if $\operatorname{Card}\left(A_{i}^{k}\right)>\operatorname{Card}\left(A_{j}^{k}\right)$. Consequently, granular world, that contains all granular hierarchies on the same domain would be defined as $\Phi=(X, \Psi, M)$ where $\mathrm{M}$ is a mapping over the hierarchies. Finally, granular universe, $\mathrm{Z}=\left(U, \Phi^{\prime}, \Delta\right)$ contains all granular worlds and consequently hierarchies where $\Phi^{\prime}=\bigcup \Phi, \Delta$ indicates communication rules amongst granular worlds and $U=\bigcup X$. Be aware that $X$ should not necessarily be defined over numerical data, but rather would be a set of granules. This will enable using multiple formal frameworks in a granular hierarchy.

We argue that, granular universe, depicts all the contextual information and any contextual information at any granulation level would be retrieved through that. It is clear that the idea of super sensors is feasible by constructing related granular hierarchy based on the directly sensed and granulated information. Potentially the granular universe would be used by both discrete and continues [4] context-aware applications. While continues context-aware applications may register their needs at the lowest level of the granular universe, discrete ones may rely on the higher levels. This is due to the trivial fact that contextual changes, hierarchically affect the granular planes, those at the lower levels would be changed even instantly in contrast to the higher level planes.

\section{CONTEXT NETWORK}

Almost all of the current context-aware applications yet developed, for context sensing rely on one of the two following methods for information gathering. In one scenario, manipulating the sensors is directly hardwired in the main application, which always leads application code being mixed with sensor handling related subcodes -that are not directly related to the logic of the application- which results in code tangling. References [11] and [16] are the examples of this kind but it must be noticed that their code is rarely reusable. Be aware that directly handling any sensor, especially regarding the diversity of sensor hardware, is not that easy and due to its nature is prone to making mistakes. Moreover, reusing the sensors and importantly simultaneously use of them by other applications is burdensome. Some other context-aware applications, on the other hand, are relied on special servers that provide them with the sensed data but force the application to deal with any server in a distinct manner rather the same way. Reference [3] is an implemented example of this approach. Here, again the application developer has to deal with each server following the rules it imposes. If context-aware applications are to be dependent on a special hardware, or following different methods to connect the servers, in essence they will not be a real context-aware application and will remain in prototypical versions.

Separating coding context-aware application on one hand and contextual information gathering with uniform and welldefined interface on the other hand, will make context-aware application development more feasible. What WSN may 
provide is yet raw numerical data, but context-aware applications need more. Toward this end, in any sensor node, SMP -in application layer- which is a management protocol that provides the software operations needed to perform administrative tasks must be equipped with the set of rules of formal framework for information granulation, i.e. set of $\left\{\left(G^{k}, A_{i}^{k}\right)\right\}$ where $i$ depends on the level of granularity the node is supposed to work in, that for sensor nodes it is usually the lowest. These rules could be apart from those that are related to data aggregation. According to the instant network needs, proper rules should be activated however, the details how such communications should take place are out of the scope of this paper. Of course, due to each sensor node's hardware capabilities and locality of the sensed signals, each node may perform basic information granulation and pass it to the sink. Based on the capabilities of sink, it may be equipped with granular plane, granular hierarchy and granular world. However, building granular universe requires more capable nodes that can see all the domains, i.e. in the sensor network hierarchy, at least one level should be added located over all sinks. Nodes in this level would be referred to as granulator (more accurately granulator-sink). However, due to some factors like the area of the sensor field, types of sensed signals, expected information granules and granulation formal frameworks other levels of granulators - over existing granulators, i.e. granulator-granulator-would be expected.

By the aforementioned granulation system, it can clearly be seen that information granules in each plane, hierarchy, world and universe is accompanied with the related rules describing its syntax and semantics. "The semantics addresses the meaning conveyed by an information granule. As being a result of some abstraction, its meaning is well-defined and practically relevant. The syntax, results directly from the formal environment in which, the granules are formed" [2]. This system provides contextual information in multiple hierarchies which are built based on formal frameworks and would be accessed by context-aware applications, without knowing how distributed sensing and transferring sensed signals and what else have taken place.

Considering context-aware application requirements to deal with context and characteristics of contextual information will reveal how context network can resolve them while providing a uniform, reliable, extensible and cost effective source of contextual information. The main requirements and characteristics drawn from [7] and [10] are briefly discussed below which proves our claim - nature and characteristics of WSN along with our view toward contextual information through granular computing has empowered context network to address these requirements and characteristics.

\section{A. Context-Aware Application Requirements to Deal} with Context [7]

Separation of concerns: Separating acquiring contextual information from how it is used, will allow applications using contextual information to be developed independent of the detail of acquiring contextual information.
Context interpretation: Numerical data gathered from the environment, must primarily be interpreted to be useful for context-aware applications.

Transparent, distributed communication: Most likely, sensors are distributed in the environment moreover different applications running on different machines may need them. The fact that sensing, processing collected data and communication is distributed should be transparent to them.

Constant availability of context acquisition: Since it is not known a priori when an application requires contextual information and moreover different application may need the same contextual information, the system that acquire contextual information must be executing independently from the applications that may use them.

\section{B. Characteristics of Contextual Data [10]}

Heterogeneity and Complexity: Heterogeneous real world situations involve many types of entities which must be handled moreover contextual information are almost very far away from simple numerical data.

Distribution: Entities to be monitored are spread all over the world.

Data Quality: Contextual information represents imperfect views on the real world.

Dynamics, mutability and unavailability: Environment is in permanent change, but not all changes will change the context, on the other hand the type of contextual information itself may change and may not be immediately visible by existing sensors.

\section{CONCLUSION}

In a simple scenario, having applications automatically adapt to their context will reduce user distractions. Such applications must be fed with reliable, applicable and proper contextual information. Providing an infrastructure that provides contextual information that would be used simultaneously by different applications with different needs through well-defined interface, facilitates developing contextaware applications. Being cost-effective, self-organizing, robust and scalable on one hand and the distributed and continuum form of gathering information from even hostile environment has made WSN such attractive that the technology has found its way in many fields, from military, security surveillance, habitat monitoring, building monitoring, environmental applications like flood and forest fire detection to human health care, home automation. It is easily possible to deploy different types of sensors for different sensing tasks, even all integrated on the same board and even deploying different kinds of sensors for performing the same sensing task to enhance the quality of sensed data. But by all means, the sensed data by WSN is almost raw. Context network, through reconsidering contextual information definition in terms of granular computing and equipping sensor nodes' architecture with another management protocol that encompasses required formal frameworks for information granulation, plus extending hierarchical structure of the network with granulators, provides the suitable source of contextual information that would be readily used by context aware applications. Due to the formal frameworks, based on which information granulation is 
performed a well-defined interface is a consequence and then e.g. CIS [13] -with modifications- would be applicable.

\section{REFERENCES}

[1] I.F. Akyildiz, Su, W., Sanakarasubramaniam Y., Cayirci E., "Wireless sensor networks: a survey", Computer Networks 38, 393-422, 2002.

[2] A. Bargiela, W. Pedrycz , "Granular Computing", Kluwer Academic Publisher, 2002.

[3] M. Bauer, T. Heiber, G. Kortuem, Z. Segall, "A collaborative wearable system with remote sensing". Proceedings of the 2 nd Int. Symposium on Wearable Computers (ISWC'98), 10-17. Los Alamitos, CA: IEEE, 1998.

[4] P.J Brown, J.D. Bovey, X. Chen, "Context-Aware Applications: from the Laboratory to the Marketplace", IEEE Personal Communications, 4(5), pp. 58-64, 1997.

[5] N. Davies, K. Mitchell, K. Cheverest, G. Blair, "Developing a Context Sensitive Tourist Guide", First Workshop on Human Computer Interaction with Mobile Devices, GIST Technical Report G98-1, 1998

[6] A.K. Dey, G.D. Abowd, "Toward a better understanding of context and context-awareness." GVU Technical Report GIT-GVU-99-22, College of Computing, Georgia Institute of Technology, 1999.

[7] A.K. Dey, G. D. Abowd, D. Salber, "A conceptual framework and toolkit for supporting the rapid prototyping of context aware applications", Human Computer Interaction (HCI) Journal, Vol. 16(2-4), pp 97-166, 2001.

[8] A.K. Dey, D. Salber, G.D. Abowd, M. Futakawa, "The Conference Assistant: Combining context-awareness with wearable computing". 3rd International Symposium on Wearable Computers, San Francisco, California, pp. 21-28, 1999.

[9] L. J. Guibas, "Sensing, tracking, and reasoning with relations," IEEE Signal Processing Magazine, pp73-85, March 2002.

[10] K. Goslar, A. Schill, "Modeling Contextual Information Using Active Data Structures". EDBT Workshops, pp.325-334, 2004.
[11] B. L. Harrison, K. P. Fishkin, A. Gujar, C. Mochon, , R. Want, "Squeeze me, hold me, tilt me! An exploration of manipulative user interfaces." Proc. of the CHI'98 Conf. on Human Factors in Computer Systems, New York, ACM Press, 1998.

[12] C. Intanagonwiwat, R. Govindan, D. Estrin, "Directed diffusion: a scalable and robust communication paradigm for sensor networks". Proc. of the Sixth Annual Int. Conf. on Mobile Computing and Networks (MobiCOM 2000), Boston, 2000

[13] G. Judd, P. Steenkiste, "Providing Contextual Information to Pervasive Computing Applications", Proc. of the 1st IEEE Int. Conf. on Pervasive Computing and Communications, pp.133-142, 2003.

[14] T. Kumar, F. Zhao, D. Shepherd, "Collaborative Signal and Information Processing in Microsensor Networks," IEEE Signal Processing Magazine, vol. 19, no. 2, pp. 13-14, 2002.

[15] T.P. Moran, P. Dourish, "Introduction to This Special Issue on ContextAware Computing", Human-Computer Interaction, 2001, Vol. 16, No. 2 , 3 \& 4, Pages 87-95.

[16] J. Rekimoto, "Tilting operations for small screen interfaces", Proceedings of the 9th Annual ACM Symposium on User Interface Software and Technology (UIST'96), 167-168. NewYork, NY: ACM Press, 1996.

[17] P. Rentala, R. Musunuri, S. Gandham, U. Saxena, "Survey on sensor networks", in Proc. of Int. Conf. on Mobile Computing and Networking, 2001.

[18] D. Salber, A.K. Dey, G.D. Abowd, "The Context Toolkit: Aiding the Development of Context-Enabled Applications", CHI'99, pp. 434-441, 1999.

[19] D. Salber, A.K. Dey, R.J. Orr, G.D. Abowd, "Designing for Ubiquitous Computing: A Case Study in Context Sensing". GVU Technical Report GIT-GVU-99-29.

[20] R. Want, A. Hopper, V. Falcao, J. Gibbons, "The Active Badge Location System". ACM Transactions on Information Systems 10(1) pp. 91-102, 1992. 